06

\title{
Экстремальная и топологическая нелинейная оптика открытых систем
}

\author{
(C) Н.Н. Розанов ${ }^{1,2,3}$, М.В. Архипов 2,4 , Р.М. Архипов ${ }^{2,3,4}$, Н.А. Веретенов ${ }^{3}$, \\ А.В. Пахомов ${ }^{2,4}$, С.В. Федоров ${ }^{2,3}$ \\ ${ }^{1}$ Государственный оптический институт им. С.И. Вавилова, \\ 199053 Санкт-Петербург, Россия \\ ${ }^{2}$ Университет ИТМО, \\ 197101 Санкт-Петербург, Россия \\ ${ }^{3}$ Физико-технический институт им. А.Ф. Иофрфе, \\ 194021 Санкт-Петербург, Россия \\ ${ }^{4}$ Санкт-Петербургский государственный университет, \\ 199034 Санкт-Петербург, Россия \\ e-mail: nnrosanov@mail.ru
}

Поступила в редакцию 25.02.2019 г.

В окончательной редакции 25.02.2019 г.

Принята к публикации 15.03.2019 г.

Представлен обзор результатов исследований по теории оптических волновых пакетов с экстремальными свойствами по отношению к управляемой форме импульсов или к сложности внутренней структуры пучков импульсов излучения. При этом особое внимание уделяется проявлениям диссипативных эффектов притока и оттока электромагнитной энергии. Именно эти факторы приводят к своеобразным правилам сохранения чисто электромагнитных величин в диссипативных средах, в которых электромагнитная энергия необратимо убывает в случае поглощения и возрастает при усилении. Эти правила накладывают определенные запреты на возможности преобразования формы импульсов и позволяют качественно описать их эволюцию. Показано повышение эффективности воздействия на классические и квантовые микрообъекты униполярных или квазиуниполярных импульсов излучения и обсуждены возможные пути формирования таких импульсов. Для объемных лазерных сред с насыщающимся поглощением описаны топологические свойства локализованных структур излучения и их трансформация при плавном изменении параметров среды (уровня накачки). Сохранение топологической структуры при изменении параметров, не выходящем за пределы области устойчивости, предоставляет возможность их различения при кодировании информации топологическими солитонами рассмотренного типа.

Ключевые слова: Предельно короткие оптические импульсы, однополярные импульсы, диссипативные системы, топологические лазерные солитоны.

DOI: $10.21883 /$ OS.2019.07.47934.80-19

\section{Введение}

Прогресс лазерной физики и техники, подтвержденный последней нобелевской премией по физике [1], позволяет генерировать пакеты излучения с предельно короткой длительностью и предельно высокой напряженностью поля [2], а также управлять топологией света - внутренней структурой генерируемых световых пакетов [3]. Естественное стремление к дальнейшему продвижению в область экстремальных значений параметров - большей концентрации излучения и большей сложности внутренней структуры его пакетов - ставит новые принципиальные вопросы уже к самой возможности получения таких структур света и их совместимости с физическими ограничениями.

Целью настоящего обзора служит анализ преимущественно двух таких вопросов. Первый из них: насколько возможно уменьшать длительность импульсов излучения, реальны ли „субцикловые“ и униполярные импульсы, и если реальны, то каков их потенциал? Второй вопрос применим уже к „обычным“ пакетам излучения, содержащим значительное число оптических колебаний. Возможно ли формирование и устойчивое распространение пакетов излучения со сложной внутренней структурой (топологией), что могло бы позволить кодировать и передавать информацию с большей надежностью? Отметим, что для ответа на оба эти вопроса необходим учет такой принципиальной особенности лазерных систем, как их диссипативность или открытость, т.е. наличие релаксационных процессов и баланса поглощения и усиления излучения в системе.

Ответу на первый из этих вопросов посвящены три последующих раздела настоящего обзора. В следующем разделе мы обсудим ограничения, которые накладывают вызванные диссипативностью правила сохранения электродинамических интегральных величин. Затем анализируются особенности воздействия предельно коротких импульсов на простейшие классические и квантовые 
объекты с выявлением более высокой эффективности квазиуниполярных импульсов. Сами методы формирования таких импульсов рассматриваются в последнем разделе этого блока. Результаты исследований по второму вопросу излагаются в едином разделе, в котором представляется внутренняя структура разнообразных топологических диссипативных солитонов в лазерной среде с быстрым насыщающимся поглощением. Обзор завершается заключительными соображениями о потенциале экстремальной и топологической лазерной оптики.

\section{Ограничения на характеристики предельно коротких импульсов}

Запреты на реализацию каких-либо процессов могут следовать из законов сохранения. Для открытых (диссипативных) сред возможность сохранения чисто электродинамических характеристик может вызвать сомнения, так как в результате поглощения излучения в реальных средах при длительной эволюции вся энергия электромагнитного излучения переходит в среду (в тепловую энергию) и „поля не остается“. Тем не менее в работах [4-7] были найдены и изучены несколько сохраняющихся чисто электромагнитных величин неэнергетического типа (для дискретных систем с трением возможно также введение функции Лагранжа и соответствующих законов сохранения другого типа, см. [8,9] и приведенную там литературу). Ниже приводится краткое пояснение, а более детальное изложение можно найти в обзоре [10].

Привлечем одно из эволюционных уравнений Максвелла электродинамики сплошных сред для напряженности электрического поля $\mathbf{E}$ и магнитной индукции $\mathbf{B}$ [11]:

$$
\operatorname{rot} \mathbf{E}=-\frac{1}{c} \frac{\partial \mathbf{B}}{\partial t}
$$

Здесь $c$ - скорость света в вакууме и $t-$ время. Считаем, что пакет излучения локализован в начальный момент в конечной пространственной области и поля $\mathbf{E}$ и В достаточно быстро убывают на периферии. Тогда после интегрирования (1) по всему пространству найдем

$$
\frac{d}{d t} \mathbf{V}_{B}=0
$$

где сохраняющаяся величина $\mathbf{V}_{B}=\iiint_{V} \mathbf{B} d \mathbf{r}-$ „объем магнитного поля“".

Теперь используем конечность длительности пакета, т. е. достаточно быстрое убывание поля на переднем и заднем временных фронтах пакета. Проинтегрировав (1) по времени в бесконечных пределах, получим

$$
\operatorname{rot} \mathbf{S}_{E}=0
$$

где введена ,электрическая площадь “ импульса $\mathbf{S}_{E}=\int_{t=-\infty}^{+\infty} \mathbf{E} d t$. Эта величина имеет смысл импульса электрической части силы Лоренца, действующей на единичный (пробный) электрический заряд и вызывающий, в соответствии со вторым законом Ньютона, изменение его механического импульса [12]. В схемах c одномерной геометрией (существенна зависимость полей только от одной пространственной координаты $z$ и от времени $t$ ) (3) также принимает вид правила сохранения:

$$
\frac{d}{d z} \mathbf{S}_{E}=0
$$

Отметим, что при выводе этих точных, в рамках уравнений Максвелла, соотношений нам было достаточно использовать только одно из этих уравнений. Аналогичная ситуация с правилами сохранения имеет место в акустике применительно к распространению звуковых пакетов в вязких газах или жидкостях [13].

Обратимся теперь к некоторым следствиям правил сохранения на примере (4), которые существенны применительно к предельно коротким импульсам. Пусть реализуются условия, при которых распространение пакета волн преимущественно однонаправленное, т.е. рассеяние излучения назад неэффективно. Тогда мы видим, что электрическая площадь импульса не меняется безотносительно к тому, обладает среда поглощением или усилением. Например, распространение импульса в поглощающей среде приводит к уменьшению характерной пиковой амплитуды поля в $n$ раз, но при этом также в $n$ раз увеличивается характерная длительность импульса. В результате электрическая площадь импульса сохраняется, тогда как его энергия, пропорциональная квадрату амплитуды, убывает в $n$ раз. Аналогично в усиливающей среде возрастание амплитуды поля сопровождается сокращением длительности импульса, что сохраняет электрическую площадь, но ведет к возрастанию энергии импульса. Тем самым прямое увеличение электрической площади импульса в усиливающей среде оказывается невозможным.

Напомним, что сделанные выводы относятся к пакетам излучения с конечной пространственной протяженностью и длительностью и неприменимы, например, к идеальным структурам типа монохроматической волны или к лазеру с „невыключающейся“ накачкой. Кроме того, в рассмотрение не включались флуктуации и шумы, так что уровень амплитуды поля должен заметно превосходить шумовой уровень.

Следует указать, что в настоящее время нередко поднимается вопрос о возможности самого существования импульсов излучения с ненулевой электрической площадью. Сразу укажем, что уравнения Максвелла отнюдь не запрещают существование таких импульсов. Более того, например, следующее непосредственно из уравнений Максвелла одномерное волновое уравнение в вакууме допускает, согласно Даламберу, существование импульсов произвольной формы $\mathbf{E}(z, t)=\mathbf{E}(z-c t)$, движущихся со скоростью света $c$ [14]. Естественно, сюда входят и униполярные импульсы, у которых знак 
амплитуды основных поляризационных составляющих при распространении не меняется. Довод о том, что ненулевая площадь импульса отвечает ненулевой амплитуде поля с нулевой частотой, которая соответственно „не может распространяться“, безусловно, неприемлем. Об этом говорит только что приведенное решение Даламбера в виде бегущего импульса. Действительно, мы можем разложить такое решение в спектр плоских монохроматических волн вида $\exp (i k z-i \omega t)$ с различными амплитудами, где $\omega$ и $k$ - частота и волновое число парциальных волн. Для униполярных импульсов амплитуда нуль-частотной составляющей отлична от нуля. Однако скорость движения монохроматических волн (фазовая скорость) определяется не просто частотой излучения $\omega$, a ее отношением к волновому числу $k$, и это отношение равно скорости света $c$ независимо от частоты. В этом смысле и нуль-частотная составляющая, понимаемая как предел компонент разложения при $\omega \rightarrow 0$, движется с той же скоростью света. Известны и движущиеся униполярные импульсы в нелинейной среде как решения уравнений самоиндуцированной прозрачности [15].

Оставляя до следующих разделов обсуждение методов получения униполярных импульсов, отметим, что в реальной ситуации точнее говорить о квазиуниполярных импульсах, характеризуя их степенью униполярности [16]

$$
\xi(\mathbf{r})=\frac{\left|\int_{-\infty}^{+\infty} \mathbf{E}(\mathbf{r}, t) d t\right|}{\int_{-\infty}^{+\infty}|\mathbf{E}(\mathbf{r}, t)| d t} .
$$

Идеальному униполярному импульсу отвечает $\xi=1$, а идеальному биполярному $\xi=0$. Реальные импульсы обладают промежуточными значениями степени униполярности, причем для квазиуниполярных импульсов $1-\xi \ll 1$.

\section{Взаимодействие квазиуниполярных импульсов с микрообъектами}

По-видимому, очевидным является то обстоятельство, что воздействие на заряженную классическую частицу униполярных электромагнитных импульсов значительно эффективней, чем стандартных импульсов, с многократным изменением направления напряженности поля, когда поле излучения на различных оптических полупериодах то ускоряет, то замедляет заряд. Действительно, для нерелятивистского движения электрического заряда $q$ (скорость движения много меньше скорости света) основной в силе Лоренца является электрическая составляющая $\mathbf{F}=q \mathbf{E}$. Тогда в соответствии со вторым законом механики Ньютона изменение механического импульса частицы

$$
\mathbf{p}-\mathbf{p}_{0}=\mathbf{N}
$$

где $\mathbf{N}=\int_{t=-\infty}^{+\infty} \mathbf{F} d t=q \mathbf{S}_{E}-$ механический момент (импульс) силы Лоренца, а р и $\mathbf{p}_{0}-$ конечный и начальный импульсы частицы [12].

Несколько подробней возможность прямого ускорения релятивистской (но не ультрарелятивистской, когда существенными становятся квантово-электродинамические эффекты и эффекты радиационного торможения [14]) рассматривалась в [17]. При нерелятивистских импульсах частицы $p=|\mathbf{p}| \ll m c$, где $m$ - масса заряженной частицы, ускорение, действительно, определяется электрической площадью электромагнитного импульса $S=|\mathbf{S}|$. Кроме того, для релятивистской частицы с зарядом $q$ и униполярного импульса излучения прямоугольной формы имеет место следующее выражение для энергии исходно неподвижной частицы:

$$
E=m c^{2}+\frac{(q S)^{2}}{2 m} .
$$

То есть и в этом случае эффективность воздействия импульса излучения определяется его электрической площадью.

Обратимся теперь к вопросу о воздействии предельно коротких импульсов излучения на простейшие квантовые объекты. Используем приближение Мигдала мгновенного возмущения [18], которое применимо и к рассматриваемой задаче (см. [19] и приведенную там литературу; здесь мы будем следовать более простому изложению [20]). Исходим из нерелятивистского уравнения Шредингера (тем самым спиновые эффекты здесь не рассматриваются) для волновой функции $\Psi$, используя атомные единицы:

$$
i \frac{\partial \Psi}{\partial t}=H_{0} \Psi+V \Psi
$$

Здесь $H_{0}$ - гамильтониан свободного объекта (в отсутствие электромагнитного импульса), а $V$ - потенциал взаимодействия объекта с излучением. Если импульс излучения достаточно интенсивен и короток (для атома - короче обратных атомных частот), то во время его воздействия на объект основным членом в уравнении Шредингера является последний, и волновая функция объекта после окончания импульса $\Psi_{t=+0}$ выражается через волновую функцию до воздействия импульса $\Psi_{t=-0}$ следующим образом:

$$
\Psi_{t=+0}=\exp \left(-i \int V d t\right) \Psi_{t=-0} .
$$

Знание волновой функции позволяет найти среднее значение импульса:

$$
\langle\mathbf{p}\rangle=\langle\Psi|\mathbf{p}| \Psi\rangle=-i \int \Psi^{*} \nabla \Psi d \mathbf{r} .
$$


До начала действия импульса излучения среднее значение механического импульса

$$
\left\langle\mathbf{p}_{t=-0}\right\rangle=\left\langle\Psi_{t=-0}|\mathbf{p}| \Psi_{t=-0}\right\rangle=-i \int \Psi_{t=-0}^{*} \nabla \Psi_{t=-0} d \mathbf{r} .
$$

При указанных выше условиях волновая функция после окончания импульса излучения имеет вид произведения начальной волновой функции $\Psi_{t=-0}$ и волновой функции плоской волны с импульсом $-\mathbf{S}_{E}$ :

$$
\Psi_{t=+0}=\exp \left(-i \mathbf{S}_{E} \mathbf{r}\right) \Psi_{t=-0} .
$$

Тогда среднее значение импульса частицы с отрицательным (единичным по модулю) электрическим зарядом после окончания действия импульса излучения

$$
\begin{aligned}
\left\langle\mathbf{p}_{t=+0}\right\rangle & =-i \int \Psi_{t=-0}^{*}(\mathbf{r})\left[-i \mathbf{S}_{E} \Psi_{t=-0}(\mathbf{r})+\nabla \Psi_{t=-0}(\mathbf{r})\right] d \mathbf{r} \\
& =\left\langle\mathbf{p}_{t=-0}\right\rangle-\mathbf{S}_{E} .
\end{aligned}
$$

Это соотношение с учетом отрицательности электрического заряда частицы служит квантовым аналогом классического закона изменения или сохранения импульса (6).

В атомных системах с дискретным и сплошным спектром энергетических уровней действие импульса излучения проявляется также в переходах между уровнями и ионизации атомов. В указанных выше приближениях (высокие пиковые амплитуды поля и короткие длительности импульса излучения) вероятность сохранения исходного основного состояния после воздействия импульса для атома водорода имеет вид [20]

$$
w_{0}=\frac{1}{\left(1+\frac{S_{E}^{2}}{4}\right)^{4}} .
$$

Поэтому атом остается в основном состоянии после взаимодействия со стандартными биполярными импульсами с нулевой электрической площадью (с учетом указанных приближений), и эта вероятность быстро убывает с ростом этой площади $\left(w_{0} \approx\left(2 / S_{E}\right)^{8}\right.$ при $\left.S_{E}^{2} \gg 1\right)$. Аналогичный вывод об эффективности воздействия квазиуниполярных импульсов на квантовые системы следует и из анализа [21] модели квантового гармонического осциллятора и родственных многоуровневых квантовых систем.

Изложенные в настоящем разделе результаты показывают, что эффективность воздействия предельно коротких импульсов на различные микрообъекты в широком диапазоне условий определяется не столько энергией импульса излучения, сколько электрической площадью этого импульса. Поэтому перспективной представляется разработка методов генерации квазиуниполярных импульсов. Такой вывод [17] существен применительно к проектированию сверхмощных лазерных систем „экстремального света“ [2]. Некоторые возможности формирования квазиуниполярных импульсов рассматриваются в следующем разделе.

\section{Методы формирования и управления формой квазиуниполярных импульсов}

Прежде всего необходимо отметить существенный прогресс в генерации предельно коротких импульсов излучения [22-24]. Помимо уже упоминавшегося приложения к ускорению заряженных частиц, такие импульсы открывают возможность изучения и контроля динамики сверхбыстрых процессов в физических, химических и биологических явлениях, генерации яркого рентгеновского излучения и т.д. [25]. Ряд аспектов теории распространения предельно коротких импульсов излучения рассматривается, помимо [16], в обзорах [26-31]. Отметим, что теория таких импульсов имеет свою специфику; в частности, по нашему мнению, при принятии различных упрощающих предположений необходимо учитывать их совместимость с такими точными следствиями уравнений Максвелла, как приведенные выше правила сохранения. По-видимому, первые теоретические исследования униполярных импульсов излучения, возникающих при движении заряженных частиц, были выполнены в работах [32-34]. Недавно в работе [35], по мнению ее авторов, впервые экспериментально были получены униполярные импульсы при дифракции пучка релятивистских электронов на краю металлического экрана. Полуцикловые импульсы аттосекундной длительности в оптическом диапазоне получены экспериментально в [36]. Некоторые другие методы получения полуцикловых импульсов аттосекундной длительности обсуждаются в [37,38]. Отметим также возможность экспериментального получения полуцикловых импульсов в терагерцовом диапазоне частот [39-42].

Напомним, что сохранение электрической площади в одномерных схемах запрещает преобразование исходного биполярного импульса с нулевой электрической площадью в однополярный. Однако возможно разбиение такого импульса на два с противоположной полярностью и различным направлением распространения или задержкой по времени. Последний пример иллюстрируют результаты моделирования распространения исходного биполярного фемтосекундного лазерного импульса в среде с резонансным нелинейным усилением и поглощением излучения [43]. В свою очередь, униполярный импульс с большой электрической площадью может распадаться в резонансной нелинейной среде на субимпульсы с меньшей площадью [44]. В [45] предложен также простой метод генерации субциклового импульса при отражении одиночного одноциклового импульса от тонкого слоя металла или диэлектрика.

Среди других возможностей генерации униполярных или квазиуниполярных импульсов излучения следует упомянуть формирование таких импульсов при распространении коротких импульсов в комбинационноактивной среде [46,47], средах с квадратичной нелинейностью [48-50] и в средах с другими типами нелинейности (см. также указанные выше обзоры [16, 26-29]). 
В последнее время появился ряд работ, в которых описываются возможности получения квазиуниполярных импульсов при возбуждении нелинейных сред световым пятном, движущимся со сверхсветовой скоростью [51-57]. Такое пятно, возникающее при наклонном падении импульса с плоским волновым фронтом на экран, а также световой зайчик от быстро вращающегося по кругу источника излучения могут вызывать генерацию импульсов с длительностью порядка периода колебаний поля. Предложенные методы могут использоваться для получения терагерцовых импульсов необычной формы - прямоугольной, треугольной [58]. Рассмотрим кратко основную идею предложенных методов. Для простоты будем считать, что среда состоит из нелинейных осцилляторов с частотой собственных колебаний $\omega_{0}$. Будем считать, что среда обладает квадратично-нелинейной связью с полем накачки $E$, т.е. динамика поляризации среды $P$ описывается уравнением следующего вида [54,55]:

$$
\ddot{P}+\gamma \dot{P}+\omega_{0}^{2} P=g_{0} E^{2} .
$$

Здесь $\gamma$ - скорость затухания осциллятора и $g_{0}-$ коэффициент нелинейности. Физическим примером подобной среды являются комбинационно-активные среды с двумя типами связанных осцилляторов - электронного (высокочастотного) и ядерного (низкочастотного) с сильно отличающимися резонансными частотами. На среду падают два идентичных импульса с временной задержкой (рис. 1,a). Предполагаем, что каждый из них имеет длительность много меньше периода собственных колебаний среды $\left(\omega_{0} \tau \ll 1\right)$. В случае слабого затухания осцилляторов поляризация после первого импульса описывается синусоидальной функцией времени с амплитудой, зависящей от импульсов возбуждения [55]:

$$
P(t)=\Pi_{1} \sin \omega_{0} t, \Pi_{1} \equiv \frac{g_{0}}{\omega_{0}} \int_{-\infty}^{+\infty} E^{2}(t) \cos \left(\omega_{0} t\right) d t
$$

Если задержка между импульсами равна половине периода колебаний осцилляторов среды $T_{0} / 2\left(\omega_{0}=2 \pi / T_{0}\right)$, то второй импульс останавливает осцилляции, вызванные первым импульсом. В результате смещение осциллятора отображается полуволной колебаний (половиной периода синусоиды, рис. $1, b)$.

Поле излучения в дальней зоне будет пропорционально второй производной от полуволны, изображенной на рис. 1,c. Оно также будет содержать полуволну и хвосты противоположной полярности в моменты старта и остановки колебаний. Эти хвосты могут быть подавлены фильтром. Таким способом можно получить квазиуниполярные импульсы полуцикловой длительности.

Излучение, отображенное на рис. 1, 1 , испускается единичным осциллятором. Для получения поля от их ансамбля естественно рассматривать тонкий слой нелинейной среды, пренебрегая эффектами распространения
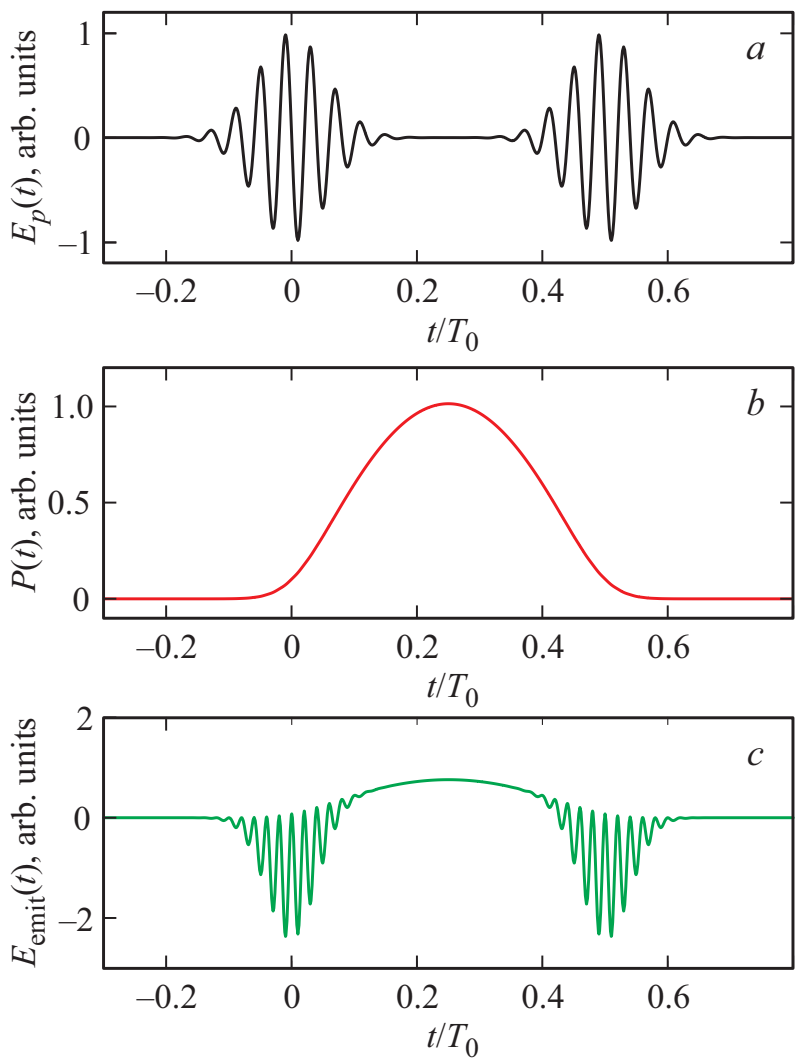

Рис. 1. Импульсы возбуждения с периодом $T_{p}=T_{0} / 2(a)$, поляризация среды $P(t)$ - полуволна синусоиды $(b)$ и поле излучения, пропорциональное второй производной поляризации $(c)$.

в нем [51-57]. Если ввести задержку в распространение полуволн от различных участков среды в точку наблюдения, то, как показывает анализ, возможно получить квазиуниполярные импульсы различной (управляемой) формы - прямоугольной, треугольной и т.д. Данная задержка может быть реализована различными способами. Например, как показано в [51,52], излучение квазиуниполярных импульсов происходит при падении двух фемтосекундных импульсов накачки с периодом $T_{0} / 2 \mathrm{c}$ плоским волновым фронтом под углом $\beta$ на систему нелинейных резонансных частиц. В этом случае область пересечения фронта с экраном перемещается вдоль экрана со сверхсветовой скоростью $v=c / \sin \beta>c$ [59]. Как известно, такой сверхсветовой зайчик может являться источником излучения Вавилова-Черенкова [59-62]. При этом полуволны будут приходить в точку наблюдения с некоторой задержкой друг относительно друга. В результате в точке наблюдения может быть сформирован квазиуниполярный импульс почти прямоугольной формы.

Аналогичная ситуация может возникать, если осцилляторы среды расположены по кругу радиусом $R[53,56,57]$. В этом случае можно получить генерацию предельно коротких импульсов, если систе- 


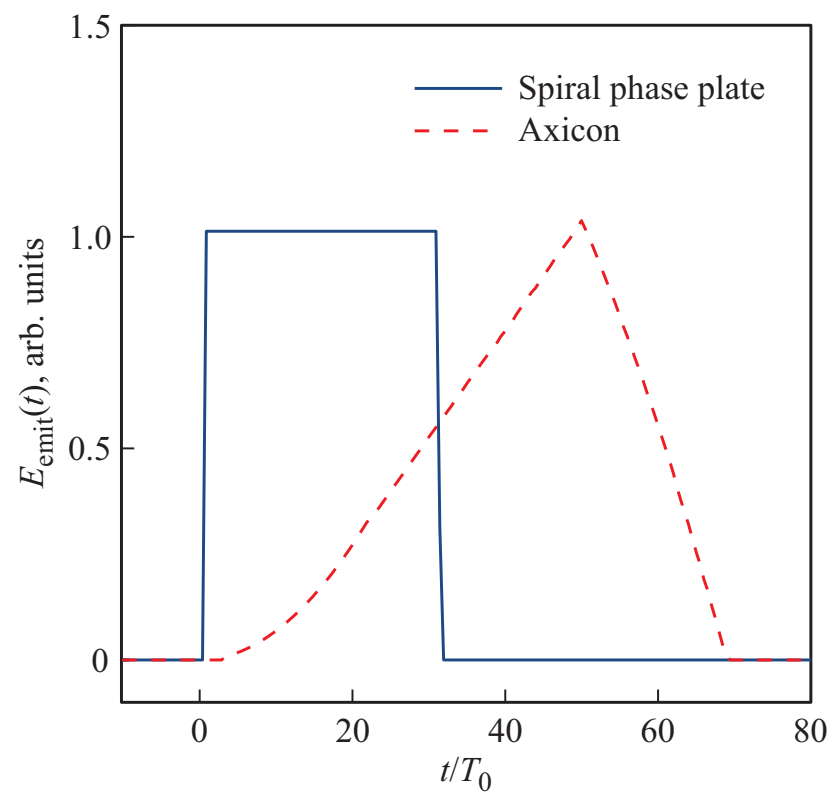

Рис. 2. Пример временного профиля терагерцовых импульсов генерации в нелинейной среде с помощью дифракционных оптических элементов - спиральной фазовой пластинки (сплошная синяя линия) и аксикона (красная штриховая линия).

ма возбуждается двумя световыми зайчиками. Зайчики должны равномерно вращаться по кругу с постоянной скоростью $v$, такой, что каждая точка круга возбуждается двумя световыми пятнышками (импульсами), приходящими через интервал времени $T_{p}=T_{0} / 2$. Это обеспечит генерацию излучения с временным профилем в виде полуволны на рис. 1 [53]. Быстро вращающийся по кругу световой зайчик может быть создан с помощью дефлекторов лазерного излучения. Возможность получения предельно коротких импульсов (ПКИ) в таких условиях изучалась в [57].

Для создания задержки в приходе вторичных волн в точку наблюдения можно использовать дифракционные оптические элементы. В качестве такого элемента может выступать, например, пластинка из прозрачного материала, толщина которой линейно нарастает с полярным углом $\varphi$, треугольный клин, толщина которого растет в направлении распространения волнового фронта, и т.д. Возможность получения квазиуниполярных терагерцовых импульсов различной контролируемой формы (прямоугольной, треугольной) с помощью дифракционных оптических элементов рассматривалась в [58], см. рис. 2.

В заключение этого раздела укажем на возможность генерации из шума и усиления низкочастотных квазиуниполярных импульсов в резонаторе с колеблющимися зеркалами за счет динамического эффекта Казимиpa [63-65].

Анализ показывает, что рассмотренные выше методы позволяют генерировать квазиуниполярные импульсы в широком спектральном диапазоне, включая терагерцовую область. Ограничением применимости представлен- ного анализа служит рассмотрение в большинстве случаев одномерного распространения, что можно оправдать либо для волноводных схем, либо для объемных сред при ограниченных длинах распространения.

\section{Топологические лазерные солитоны}

Теперь мы обратимся к возможности формирования и устойчивого распространения максимально локализованных (по всем пространственным измерениям) пакетов излучения, которые обладали бы сложной и управляемой внутренней структурой. При этом мы ограничимся рассмотрением только лазерных систем с диссипацией - притоком и оттоком энергии в форме некогерентной накачки и нелинейных потерь излучения. Будем здесь также считать отклик среды быстрым среда мгновенно следует за полем, не обладая тем самым самостоятельными степенями свободы. В отличие от предыдущих разделов мы будем полагать, что излучение близко к плоской монохроматической волне, когда оправдано приближение медленно меняющейся комплексной огибающей поля $E(\mathbf{r}, t)$. При этом полная электрическая напряженность $\mathbf{E}$ представляется в виде

$$
\mathbf{E}(\mathbf{r}, t)=\operatorname{Re}\left[E(\mathbf{r}, t) \mathbf{e}_{x} \exp \left(i k_{0} z-i \omega_{0} t\right)\right],
$$

где $k_{0}$ - волновое число излучения в линейной среде, отвечающее несущей частоте $\omega_{0}$, и $\mathbf{e}_{x}-$ единичный вектор, определяющий поляризацию излучения; последнюю будем считать фиксированной и линейной. В указанном приближении управляющее уравнение для огибающей поля имеет вид (безразмерная форма)

$$
\frac{\partial E}{\partial \xi}=\sum_{n=1}^{D} c_{n} \frac{\partial^{2} E}{\partial x_{n}^{2}}+f\left(|E|^{2}\right) E .
$$

Здесь $D$ - геометрическая размерность схемы. Одномерные и двумерные схемы реализуются в широкоапертурных лазерах с насыщающимся поглощением, в них эволюционная переменная $\xi=t$ (время), а $x_{1,2}-$ поперечные декартовы координаты (дополнительно используется приближение среднего поля с усреднением огибающей по длине резонатора [66]). Трехмерная схема $(D=3)$ представляет объемную среду с насыщающимися усилением и поглощением или лазер с такой средой и достаточно большой длиной резонатора, для нее $\xi=z$, $x_{1,2}$ имеют прежний смысл, а $x_{3}=\tau=t-z / v_{g}-$ время в сопутствующей системе координат, движущейся вдоль оси $z$ с групповой скоростью $v_{g}$. Многие аспекты теории таких схем изложены в [6], а для родственных схем в недавнем обзоре [67] и цитированной там литературе, поэтому здесь мы остановимся более подробно на полученных недавно результатах по топологическим лазерным солитонам [68-72]. Члены (18) с коэффициентами $c_{n}=i+d_{n}, d_{n} \geq 0$ описывают дифракцию, дихроизм (зависимость потерь или усиления от направления распространения волны), дисперсию и конечную ширину 


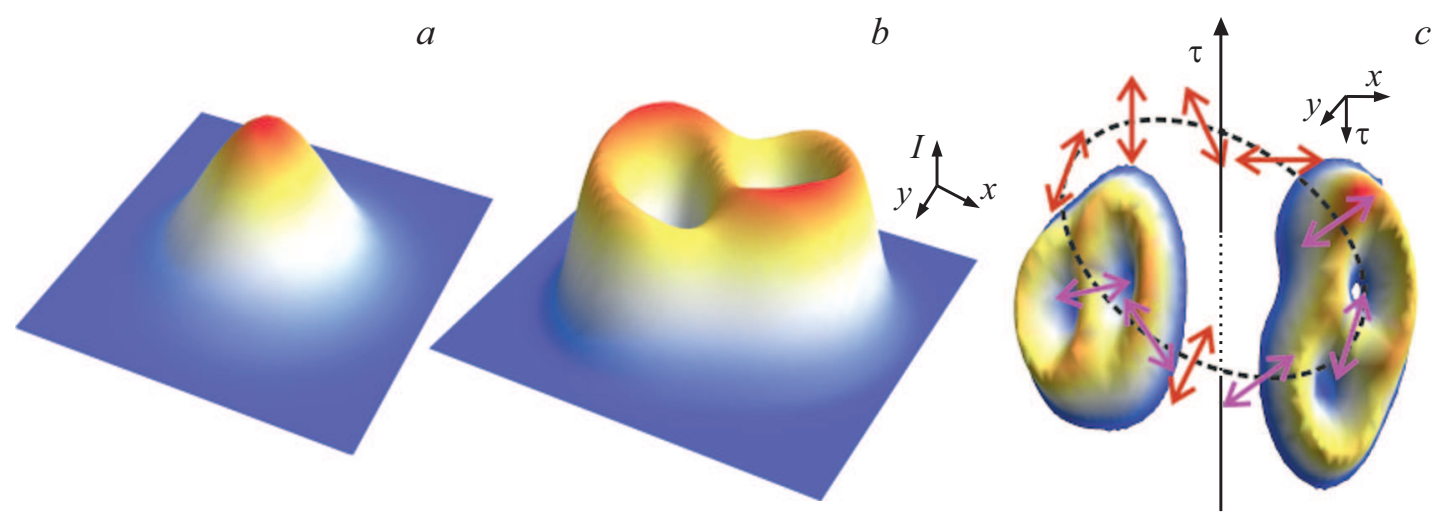

Рис. 3. Поперечные профили интенсивности двумерных структур: фундаментального солитона $(a)$ и сильно связанной пары вихревых солитонов с единичными топологическими зарядами $(b)$. Построение исходного трехмерного распределения поля вращением поля двумерной структуры $(b)$ вокруг оси $\tau$ и ее разворотом, так что центр структуры вращается по пунктирной окружности, а ориентация структуры разворачивается как показывают двунаправленные стрелки, отвечающие отрезкам между двумя центрами дислокаций волнового фронта $(c)$.

контура усиления. Функция интенсивности $I=|E|^{2}$ в общем случае комплексна, но при малых частотных расстройках она становится вещественной:

$$
f(I)=\frac{g_{0}}{1+I / \beta}-\frac{a_{0}}{1+I}-1
$$

Первый член в правой части (19) описывает насыщающееся усиление, второй - насыщающееся поглощение, а последний - нерезонансные потери $\left(g_{0}-\right.$ коэффициент линейного усиления, $a_{0}-$ коэффициент линейного резонансного поглощения, $\beta$ - отношение интенсивностей насыщения усиления и поглощения).

Важную роль в диссипативных системах играет поток „поперечный“ (дополнительный к основному, направленному вдоль $z$ энергии (вектора Пойнтинга)

$$
\mathbf{S}=\operatorname{Im}\left(E^{*} \nabla_{D} E\right)=I \nabla_{D} \Phi,
$$

где $\Phi-$ фаза излучения $\left(E=I^{1 / 2} \exp (i \Phi)\right)$. В соответствии с (20) поток направлен вдоль градиента фазы и пропорционален интенсивности излучения.

Естественно, что по мере увеличения геометрической размерности системы внутренняя структура пакетов излучения становится более разнообразной. Для дальнейшего в случае одномерных локализованных структур диссипативных солитонов - нам будет важна лишь связь движения структур в целом с их симметрией (симметрией интенсивности и потока энергии). При наличии такой симметрии структура не движется в поперечном направлении, а в отсутствие симметрии движется, если она жесткая, с постоянной линейной скоростью. В двумерной геометрии, помимо фактора симметрии, возникает возможность сингулярностей фазы - дислокаций волнового фронта. В центре дислокации интенсивность излучения обращается в нуль, а при обходе центра по замкнутому контуру фаза испытывает приращение $2 \pi m$, где целое число $m$ - топологический заряд. При этом поток энергии образует вихри в точках - центрах

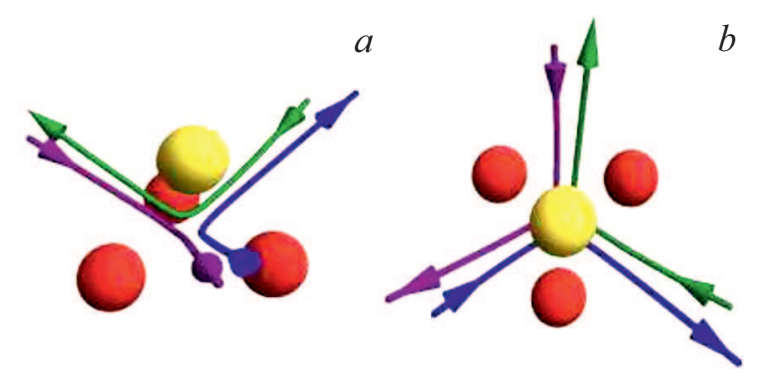

Рис. 4. Изоповерхности интенсивности и вихревые линии у пирамидального комплекса четырех слабо связанных фундаментальных солитонов, состоящего из трех синфазных и одного противофазного солитонов (в двух ракурсах). Стрелки на линиях указывают направление возрастания фазы излучения.

дислокаций. Профиль интенсивности осесимметричного фундаментального $(m=0)$ солитона показан на рис. $3, a$. На рис. 3, $b$ приведен профиль интенсивности сильно связанной пары двух вихревых солитонов с единичными топологическими зарядами; в соответствии с правилами симметрии [6] (распределения интенсивности и потока энергии имеют центральную симметрию) эта пара вращается вокруг неподвижного центра с постоянной угловой скоростью.

В трехмерной геометрии также имеются фундаментальные солитоны и их комплексы, а двумерные дислокации волнового фронта превращаются в пространственные вихревые линии. Заметим, что вихревые линии могут возникать и в комплексах фундаментальных солитонов вследствие интерференционных эффектов. Пример представлен на рис. 4 для комплекса пирамидальной формы из 4 солитонов. Такой комплекс обладает осью, проходящей через противофазный солитон и центр равностороннего треугольника, образуемого тремя синфазными солитонами. Поле симметрично относительно отражения от трех плоскостей, проходящих через ось и 
середины сторон этого треугольника. Поэтому комплекс движется равномерно и прямолинейно вдоль этой оси без вращения.

Принципиально новый класс топологических трехмерных лазерных солитонов может быть получен из двумерных [68-72], как это изображено на рис. 3, на примере двумерного комплекса, представленного на рис. $3, b$. Более точно, речь идет о приготовлении начального условия, из которого после, возможно, длительной эволюции формируется тот или иной солитон. А именно в трехмерном пространстве выбираем некоторую ось (на рис. $6, c$ это ось $\tau$ ) и задаем в проходящей через эту ось плоскости (на рисунке плоскость $x \tau$ ) и распределение поля, известное для 2D-солитона или солитонной структуры. Эта образующая 2D-структура может обладать симметрией к повороту на угол $2 \pi / N$ с целым $N$. Затем вращаем это 2D-распределение вокруг оси $\tau$ на угол $\varphi$, так что повернутая 2D-структура лежит в плоскости $\rho \tau$, где $x=\rho \cos \varphi, y=\rho \sin \varphi$. Одновременно разворачиваем 2D-структуру (если исходно она не осесимметрична) в плоскости $\rho \tau$ на угол $\theta=\varphi s / N$ с целым числом $s$ - индексом скрученности замкнутых линий, с тем чтобы после полного оборота вокруг оси $\tau$ (при $\varphi=2 \pi$ ) трехмерное распределение поля было непрерывным, а исходные двумерные дислокации волнового фронта преобразовались в замкнутые вихревые линии. Кроме того, превращаем ось $\tau$ в вихревую линию с топологическим зарядом $M$. Дальнейшая эволюция поля определяется решением управляющего уравнения (18) с функцией баланса усиления и поглощения (19). Полагая, что топология трехмерной структуры в процессе установления не меняется (с оговоркой, что исходные вихревые линии с высшим топологическим зарядом превращаются в набор линий с единичным зарядом), мы можем считать ее совпадающей с таковой для начального распределения поля и характеризовать ее указанными целочисленными индексами.

Получающееся семейство солитонов характеризуется их скелетами - наборами вихревых линий. Такие линии можно считать ориентированными в соответствии с направлением возрастания фазы. Кроме того, имеются замкнутые и незамкнутые (бесконечные) вихревые линии, так что их совокупность образует топологические тэнглы [73]. Прокомментируем найденные к настоящему времени лазерные тэнгл-солитоны, представленные на рис. 5.

Естественно было бы ожидать, что простейшей такой структурой послужит тороидальный солитон, сформированный вращением осесимметричного фундаментального солитона вокруг оси $\tau$, являющейся вихревой линией с топологическим единичным зарядом $M=1$ (сразу укажем, что вихревые линии с высшими топологическими зарядами в наших условиях всегда оказываются неустойчивыми, распадаясь на линии с единичным зарядом). Тем самым исходная 2D-структура обладает осевой симметрией распределения интенсивности и потока энергии $(N=\infty)$. Однако такая структура оказывается только метастабильной, хотя и обладающей большим временем жизни. В конце концов нарастающие возмущения разрушают симметрию и формируется асимметричный солитон с искривленной незамкнутой вихревой линией, вращающийся и прецессирующий в некотором диапазоне параметров (рис. 5,a).

Солитоны со скелетами, показанными на рис. $5, b-e$, формируются при вращении двумерного осесимметричного $(N=\infty)$ вихревого солитона с единичным зарядом и получены при различных значениях коэффициента линейного усиления $g_{0}$. По-прежнему топологический заряд исходной вихревой линии $M=1$. Мы будем называть эти солитоны „яблоками“ ввиду похожести формы распределения интенсивности. Топологически эти солитоны эквивалентны - их скелет состоит из одной незамкнутой и одной безузловой (топологически эквивалентной кругу) замкнутой, окружающей незамкнутую, вихревых линий. Тем не менее ,яблочные“ солитоны различаются как по форме вихревых линий, так и по количественным характеристикам. Так, „идеальное яблоко“, рис. 5, $b$, обладает осевой симметрией - его незамкнутая линия прямая, а замкнутая - окружность с центром в точке пересечения плоскости окружности с незамкнутой вихревой линией. Как показано на рис. 5, $c$, в этом случае замкнутая линия не окружность, но симметрична по отношению к повороту на угол $\pi / 3$. В изображенном на рис. $5, d$ случае форма замкнутой линии приобретает дополнительные угловые гармоники, а незамкнутая линия слегка изгибается. Наконец, на рис. 5, e представлена нестационарная фигура с периодическим „штопорным“ движением сильно искривленной незамкнутой вихревой линии.

Остальные иллюстрируемые рис. $5, f-j$ солитоны получались на основе комплекса двух двумерных сильно связанных вихревых солитонов с единичным топологическим зарядом. Спецификой этого комплекса служит отмеченная выше его центральная симметрия $N=2$. Различия между топологией этих солитонов обусловлены разной величиной угла разворота $\theta$ (целым числом $s)$. Так, для рис. $5, f$ разворот отсутствует $(s=0)$, и две имеющиеся для двумерного солитона дислокации волнового фронта при вращении образуют два параллельных круга.

В установившейся структуре имеются две незацепленные замкнутые безузловые вихревые линии (сжимаясь и расширяясь, они периодически проходят друг сквозь друга) и три незамкнутые вихревые линии. Число незамкнутых вихревых линий рис. $5, f-i$ три. Скелет на рис. $5, g$ имеет топологию „тривиальный узел +“; здесь знак + указывает на наличие незамкнутых вихревых линий, а тривиальность узла означает возможность превратить его плавными деформациями в трехмерном пространстве в круг при отсутствии незамкнутых вихревых линий. В этом случае разворот производился на угол $\pi$ (половину оборота, $s=-1$ ). Структура вращается и осциллирует. 


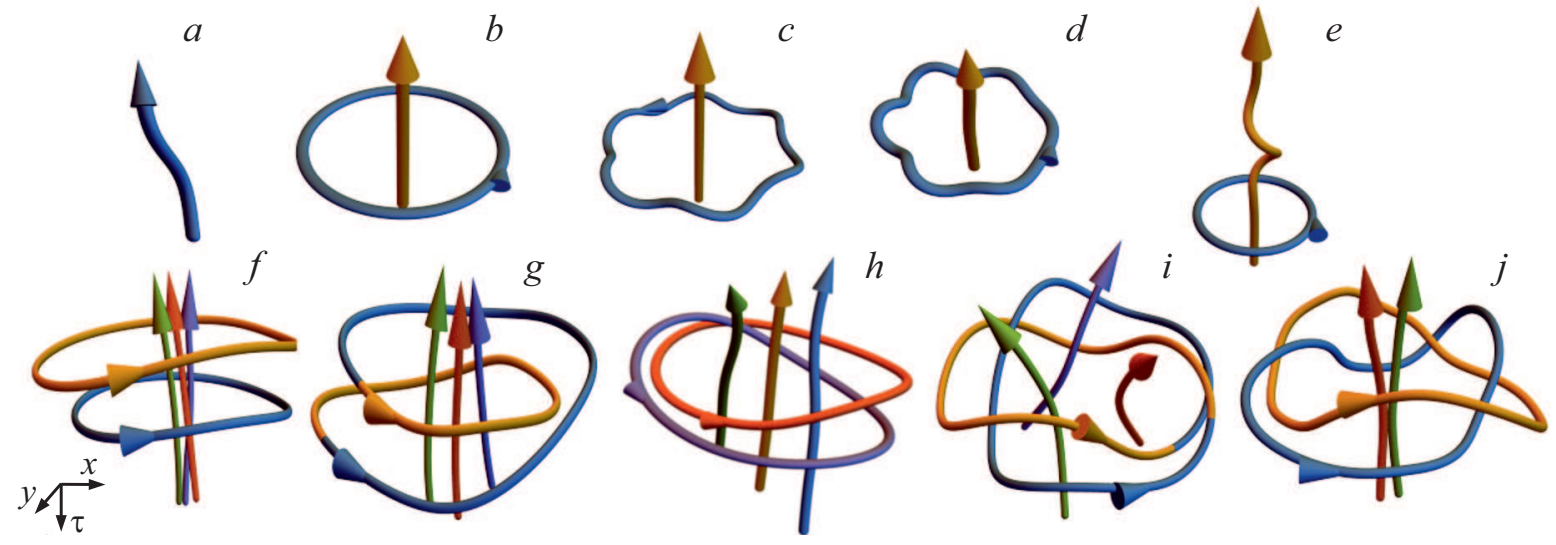

Рис. 5. Скелеты - наборы вихревых линий - тэнгл-солитонов. Число незамкнутых вихревых линий $1(a-e), 2(j)$ и $3(f-i)$. Незамкнутые линии $(a)$ отсутствуют, „прецессон“, $(b)-(e)$ - одна безузловая замкнутая, „яблоки“, $(f)$ - две безузловые незацепленные, $(g)$ - одна, тривиальный узел, $(h)$ - две безузловые, однократное зацепление Хопфа, $(i)$ - одна, узел трилистник, $(j)$ - две безузловые, с двукратным зацеплением Соломона. Индекс скрученности замкнутых линий $s=0(f),-1(g),-2(h)$, $-3(i),-4(j)$. Стрелки на вихревых линиях указывают направление возрастания фазы излучения.

Скелет на рис. 5, $h$ классифицируется как „зацепление Хопфа +“", так как включает две незамкнутые безузловые линии, однократно зацепленные. Вообще говоря, для получения такой структуры следовало бы развернуть двумерный солитон на угол $2 \pi$ (один оборот, $s=-2$ ), но в действительности в расчетах она возникла сначала другим образом, при распаде более сложной метастабильной структуры. Структура вращается и является в определенной области параметров жесткой. В случае рис. 5, $i$ мы имеем дело с единой узловой (формы трилистника, которую нельзя преобразовать в круг плавными деформациями) незамкнутой вихревой линией. Разворот двумерной структуры здесь производился на угол $3 \pi$ (полтора оборота, $s=-3$ ). Структура оказывается жесткой. Наконец, скелет на рис. $5, j$ получается разворотом образующей двумерной структуры на угол $4 \pi$ (два оборота, $s=-4$ ). В этом случае имеются две незамкнутые вихревые линии и две безузловые замкнутые, дважды зацепленные („зацепление Соломона $\left.+{ }^{\text {“6}}\right)$.

Конечно, имеется гораздо большее число неустойчивых, хотя и долго живущих метастабильных структур, которые при длительной эволюции теряют локализованность или превращаются в один из устойчивых солитонов. При этом различные солитоны сохраняют устойчивость в различных областях параметров. И здесь ввиду частичного перекрытия областей устойчивости возникают своеобразные гистерезисные явления. Они реализуются при медленном изменении контрольного параметра, например, коэффициент линейного усиления $g_{0}$ сначала плавно возрастает с выходом за границу области устойчивости, а затем медленно убывает, возвращаясь к исходному значению. Естественно, что изменения контрольного параметра вызывают изменение характеристик солитонов. Оказывается, что если при этом топология структуры (ее скелета) не меняется,
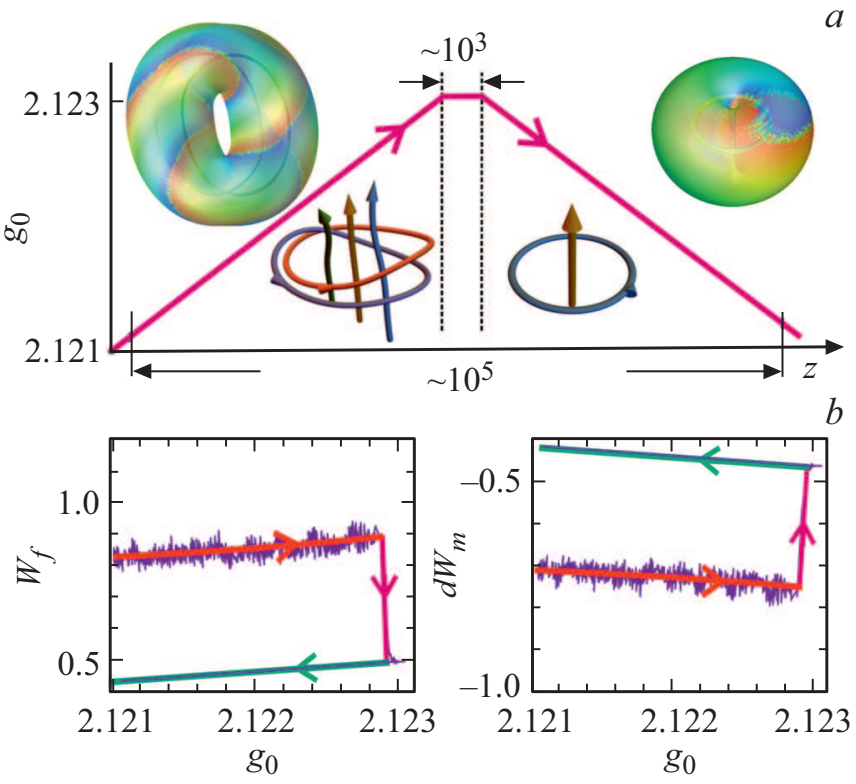

Рис. 6. Необратимый гистерезис солитона „зацепление Хопфа +““ - „яблоко“. (а) Продольное изменение коэффициента линейного усиления, на врезках показаны изоповерхности интенсивности солитонов (над линией) и их скелеты (под линией). (b) Гистерезисное изменение энергии поля (слева) и отклонения энергии среды от равновесного значения (справа) при изменении коэффициента усиления; стрелки указывают на возрастание или убывание этого коэффициента.

как это имеет место при переходах между различными типами солитона-,яблока“ (рис. 5, $b-e$ ), то гистерезис является обратимым, так что после окончания гистерезисного цикла структура солитона восстанавливается. При промежуточных же изменениях топологии гистерезис оказывается необратимым. Пример приведен на рис. 6. Если на этапе роста коэффициента усиления 
солитон был типа „зацепление Хопфа +““, то после ряда топологических реакций и трансформаций при убывании усиления он превращался в „яблоко“, устойчивое в наиболее широкой области параметров. При этом в итоге гистерезисного цикла топологическая структура солитона упрощается, энергия поля $W_{f}=\int|E|^{2} d \mathbf{r}$ убывает, а отклонение энергии среды от равновесного уровня $\delta W_{m}=\int\{f[I(\mathbf{r})]-f(0)\} d \mathbf{r}$ возрастает. Тем самым гистерезис является необратимым. Следует также оговорить, что конечная точность реальных расчетов играет роль шумов, из-за чего потеря устойчивости солитона может происходить еще до пересечения границы области его устойчивости, хотя и близко к ней.

\section{Заключение}

Приведенный обзор свидетельствует, по нашему мнению, о значительном потенциале экстремальной и топологической лазерной оптики для расширения представлений о взаимодействии пакетов излучения с микрообъектами и перспективах „тонкого“ управления пространственно-временным профилем этих пакетов и их внутренней структурой в реальных условиях, когда существенны диссипативные эффекты (приток и отток электромагнитной энергии). Представляется важным вывод о совместимости униполярных или квазиуниполярных электромагнитных импульсов с точными электродинамическими уравнениями Максвелла и реализуемости таких импульсов с управляемой формой, по крайней мере, для эффективно одномерных схем (световодная конфигурация или ограниченные трассы распространения); расширение этих представлений для существенно трехмерных схем требует дальнейших исследований. Для предельно коротких импульсов критерий их эффективности существенно зависит от задачи и в ряде важных случаев при конструировании соответствующих лазерных установок следовало бы стремиться не к большей энергии импульса, а к его большей электрической площади. Действительно, применительно к свободным заряженным частицам униполярный импульс, действуя однонаправленно, эффективней ускоряет такие частицы, чем биполярный импульс с изменением самого направления воздействия на частицы. Для более сложных объектов, обладающих резонансами, значимость последних при воздействии коротких импульсов (с длительностью короче отвечающих резонансам периодов) пропадает. Поэтому униполярные импульсы более эффективно воздействуют и на квантовые микрообъекты типа атома или молекулы.

По-видимому, можно говорить и о значительном прогрессе в развитии топологической лазерной оптики, материальная база которой обеспечивается современной нанотехнологией, голографической техникой и пространственно-временными модуляторами. Выявление особо устойчивых диссипативных (лазерных) солитонов с топологически сложной внутренней структурой предо- ставляет новые возможности кодирования информации соответствующими „иероглифами“, распознавание которых за счет анализа их топологических характеристик не затруднено проблемами масштаба или ракурса восприятия закодированных сигналов.

\section{Благодарности}

Результаты работы, относящиеся к трехмерным узловым солитонам, были получены с использованием вычислительных ресурсов суперкомпьютерного центра Санкт-Петербургского политехнического университета Петра Великого (www.scc.spbstu.ru).

\section{Финансирование работы}

Исследование накладываемых правилами сохранения ограничений на характеристики лазерных импульсов поддержано грантом Российского фонда фундаментальных исследований, грант 19-02-00312. Изучение возможности получения квазиуниполярных импульсов в нелинейных средах и их взаимодействия с резонансными средами выполнено за счет гранта Российского научного фонда (проект № 17-19-01097). Исследование топологических солитонов поддержано грантом Российского научного фонда (проект № 18-12-00075).

\section{Конфликт интересов}

Авторы заявляют, что у них нет конфликта интересов.

\section{Список литературы}

[1] Электронный ресурс. Режим доступа: https://elementy.ru/novosti_nauki/433343/ Nobelevskaya_premiya_po_fizike_2018/

[2] Электронный ресурс. Режим доступа: https://eli-laser.eu/

[3] Brasselet E., Gervinskas G., Seniutinas G., Juodkazis S. // Phys. Rev. Lett. 2013. V. 111. N 19. P. 193901.

[4] Розанов Н.Н. // Опт. и спектр. 2009. Т. 107. № 5. С. $761-$ 765; Rosanov N.N. // Opt. Spectrosc. 2009. V. 107. N 5. P. 721-725.

[5] Rosanov N.N., Kozlov V.V., Wabnitz S. // Phys. Rev. A. 2010. V. 81. N 4. P. 043815.

[6] Розанов Н.Н. Диссипативные оптические солитоны. М., Физматлит, 2011.

[7] Розанов Н.Н. // Опт. и спектр. 2015. Т. 118. № 6. С. $975-$ 976; Rosanov N.N. // Opt. Spectrosc. 2015. V. 118. N 6. P. 943-944.

[8] Бобровницкий Ю.И. // Акуст. журн. 2005. Т. 51. № 1. C. 59-67.

[9] Шалашов А.Г. // УФН. 2018. Т. 188. № 11. С. 1191-1197. Shalashov A.G. // Phys. Usp. 2018. V. 61. N 11. P. 1082-1088.

[10] Розанов Н.Н., Архипов Р.М., Архипов М.В. // УФН. 2018. T. 188. № 12. C. 1347-1353. Rosanov N.N., Arkhipov R.M., Arkhipov M.V. // Phys. Usp. 2018. V. 61. N 12. P. 1227-1233. 
[11] Ландау Л.Д., Лифиии Е.М. Электродинамика сплошных сред. М.: Наука, 1982; Landau L.D., Lifshitz E.M., Pitaevskii L.P. Electrodynamics of Continuous Media. Oxford: Butterworth-Heinemann, 1984.

[12] Розанов Н.Н. // Опт. и спектр. 2018. Т. 125. № 6. С. 818 819; Rosanov N.N. // Opt. Spectrosc. 2018. V. 125. N 6. P. $1012-1013$

[13] Розанов Н.Н. // Письма в ЖТФ. 2019. Т. 45. В. 7. С. 36-37.

[14] Ландау Л.Д., Лифшии, Е.М. Теория поля. М.: Физматлит, 1960; Landau L.D., Lifshitz E.M. The Classical Theory of Fields. Oxford: Butterworth-Heinemann, 1975.

[15] Bullough R.K., Ahmad F. // Phys. Rev. Lett. 1971. V. 27. N 6. P. 330.

[16] Архипов Р.М., Пахомов А.В., Архипов М.В., Бабушкин И., Толмачев Ю.А., Розанов Н.Н. // Письма в ЖЭТФ. 2017. T. 105. № 6. C. 388-400; Arkhipov R.M., Pakhomov A.V., Arkhipov M.V., Babushkin I., Tolmachev Yu.A., Rosanov N.N. // JETP Lett. 2017. V. 105. N 6. P. 408-418.

[17] Розанов Н.Н. // Опт. и спектр. 2019. Т. 126. № 2. С. 211213; Rosanov N.N. // Opt. Spectrosc. 2019. V. 126. N 2.

[18] Мигдал А.Б., Крайнов В.П. Приближенные методы квантовой механики. М.: Наука, 1966. 152 с.

[19] Dimitrovski D., Solov'ev E.A., Briggs J.S. // Phys. Rev. A. 2005. V. 72. N 4. P. 043411.

[20] Розанов Н.Н. // Опт. и спектр. 2018. Т. 124. № 1. С. 75-76; Rosanov N.N. // Opt. Spectrosc. 2018. V. 124. N 1. P. 72-74.

[21] Arkhipov R.M., Pakhomov A.V., Arkhipov M.V., Babushkin I., Demircan A., Morgner U., Rosanov N.N. // Opt. Lett. 2019. V. 44. N 5. P. $1202-1205$.

[22] Krausz F., Ivanov M. // Rev. Mod. Phys. 2009. V. 81. N 1. P. 163.

[23] Manzoni C., Mücke O.D., Cirmi G., Fang S., Moses J., Huang S.-W., Hong K.-H., Cerullo G., Kärtner F.X. // Laser Photonics Rev. 2015. V. 9. N 2. P. 129-171.

[24] Calegari F., Sansone G., Stagira S., Vozzi C, Nisoli M. // J. Phys. B: At. Mol. Opt. Phys. 2016. V. 49. N 6. P. 062001.

[25] Ramasesha K., Leone S.R., Neumark D.M. // Annu. Rev. Phys. Chem. 2016. V. 67. P. 41-63.

[26] Маймистов А.И. // Квант. электрон. 2000. Т. 30. № 4. C. 287-304; Maimistov A.I. // Quantum Electron. 2000. V. 30. N 4. P. 287-304.

[27] Маймистов А.И. // Квант. электрон. 2010. Т. 40. № 9. C. 756-781; Maimistov A.I. // Quantum Electron. 2010. V. 40. N 9. P. $756-781$.

[28] Leblond H., Mihalache D. // Phys. Rep. 2013. V. 523. N 2. P. 61-126.

[29] Сазонов С.В. // Вестник ИТМО. 2013. Т. 13. № 5. С. 1-21.

[30] Arkhipov R.M., Pakhomov A.V., Arkhipov M.V., Babushkin I., Tolmachev Yu.A., Rosanov N.N. // Laser Physics. 2017. V. 27. N 5. P. 053001.

[31] Архипов Р.М., Архипов М.В., Пахомов А.В., Бабушкин И., Розанов Н.Н. // Опт. и спектр. 2017. Т. 122. № 6. C. 993-999; Arkhipov R.M., Arkhipov M.V., Pakhomov A.V., Babushkin I., Rosanov N.N. // Opt. Spectrosc. 2017. V. 122. N 6. P. 949-954.

[32] Бессонов Е.Г. // ЖЭТФ. Т. 80. № 3. С. 852-858. 1981; Bessonov E.G. // Sov. Phys. JETP. 1981. V. 53. N 3. P. 433-436.

[33] Bessonov E.G. // Nucl. Instr. and Meth. A. 1991. V. 308. P. $135-139$.
[34] Бессонов Е.Г. // Квантовая электроника. 1992. Т. 19. № 1. C. 35-39; Bessonov E.G. // Sov. J. Quant. Electron. 1992. V. 22. N 1. P. 27-31.

[35] Naumenko G., Shevelev M. // J. Instrumentation. 2018. V. 13. N 5. P. C05001.

[36] Hassan M.Th., Luu T.T., Moulet A., Raskazovskaya O., Zhokhov P., Garg M., Karpowicz N., Zheltikov A.M., Pervak V., Krausz F., Goulielmakis E. // Nature. 2016. V. 530. P. 66-70.

[37] Wu H.-C., Meyer-ter-Vehn J. // Nature Photon. 2012. V. 6. P. 304-307.

[38] Xu J., Shen B., Zhang X., Shi Y., Ji L., Zhang L., Xu T., Wang W., Zhao X., Xu Z. // Sci. Rep. 2018. V. 8. P. 2669.

[39] Gao Y., Drake T., Chen Z., DeCamp M.F. // Opt. Lett. 2008. V. 33. N 23. P. 2776-2778.

[40] Reimann K. // Rep. Progr. Phys. 2007. V. 70. N 10. P. $1597-1632$.

[41] Roskos H.G., Thomson M.D., Kress M., Loeffler T. // Laser Photon. Rev. 2007. V. 1. N 4. P. 349-368.

[42] Obraztsov P.A., Kaplas T., Garnov S.V., Kuwata-Gonokami M., Obraztsov A.N., Svirko Y.P. // Sci. Rep. 2014. V. 4. P 4007.

[43] Высотина Н.В., Розанов Н.Н., Семенов В.Е. // Опт. и спектр. 2009. Т. 106. № 5. С. 793-797; Vysotina N.V., Rosanov N.N., Semenov V.E. // Opt. Spectrosc. 2009. V. 106. N 5. P. $713-717$.

[44] Архипов Р.М., Розанов Н.Н. // Опт. и спектр. 2018. Т. 124. № 5. C. 691-694; Arkhipov R.M., Rosanov N.N. // Opt. Spectrosc. 2018. V. 124. N 5. P. 726-729.

[45] Arkhipov M.V., Arkhipov R.M., Pakhomov A.V., Babushkin I., Demircan A., Morgner U., Rosanov N.N. // Opt. Lett. 2017. V. 42. N 11. P. 2189-2192.

[46] Беленов Э.М., Назаркин А.В., Прокопович И.П. // Письма в ЖЭТФ. 1992. Т. 55. № 4. С. 223-227; Belenov E.M., Nazarkin A.V., Prokopovich I.P. // JETP Letters. 1992. V. 55. N 4. P. 218-222.

[47] Беленов Э.М., Крюков П.Г., Назаркин А.В., Прокопович И.П. // ЖЭТФ. 1994. Т. 105. № 1. С. 28-42; Belenov E.M., Kryukov P.G., Nazarkin A.V., Prokopovich I.P. // JETP. 1994. V. 78. N 1. P. 15-22.

[48] Kozlov V.V., Rosanov N.N., De Angelis C., Wabnitz S. // Phys. Rev. A. 2011. V. 84. N 2. P. 023818

[49] Бугай А.Н., Сазонов С.В. // Письма в ЖЭТФ. 2010. Т. 92. № 4. C. 260-266; Bugay A.N., Sazonov S.V. // JETP Lett. 2010. V. 92. N 4. P. 232-237.

[50] Bugay A.N., Sazonov S.V. // Phys. Lett. A. 2010. V. 374. N 8. P. 1093-1096.

[51] Архипов Р.М. // Опт. и спектр. 2016. Т. 120. № 5. C. 802-806; Arkhipov R.M. // Opt. Spectrosc. 2016. V. 120. N 5. P. $756-759$.

[52] Arkhipov R.M., Arkhipov M.V., Belov P.A., Tolmachev Yu.A., Babushkin I. // Las. Phys. Lett. 2016. V. 13. N 4. P. 046001.

[53] Arkhipov R.M., Pakhomov A.V, Arkhipov M.V., Tolmachev Yu.A., Rosanov N.N., Babushkin I.V. // J. Opt. Soc. Am. B. 2016. V. 33. N 12. P. 2518-2524.

[54] Pakhomov A.V., Arkhipov R.M., Babushkin I.V., Arkhipov M.V., Rosanov N.N. // Laser Phys. Lett. 2016. V. 13. N 12. P. 126001.

[55] Pakhomov A.V., Arkhipov R.M., Babushkin I.V., Arkhipov M.V., Tolmachev Yu.A., Rosanov N.N. // Phys. Rev. A. 2017. V. 95. N 1. P. 013804. 
[56] Архипов Р.М., Жигулева Д.О., Пахомов А.В., Архипов М.В., Бабушкин И., Розанов Н.Н. // Опт. и спектр. 2018. Т. 124. № 4. С. 505-509; Arkhipov R.M., Zhiguleva D.O., Pakhomov A.V., Arkhipov M.V., Babushkin I., Rosanov N.N. // Opt. Spectrosc. 2018. V. 124. N 4. P. 536-540.

[57] Ziguleva D.O., Arkhipov R.M., Arkhipov M.V., Pakhomov A.V., Babushkin I., Rosanov N.N. // Opt. Commun. 2018. V. 424. P. $170-176$.

[58] Pakhomov A.V., Arkhipov R.M., Arkhipov M.V, Demircan A., Morgner U., Rosanov N.N., Babushkin I. // Scientific Reports. 2019. V. 9. Article number: 7444.

[59] Болотовский Б.М., Гинзбург В.Л. // УФН. 1972. Т. 106. № 4. C. 577-592; Bolotovskii B.M., Ginzburg V.L. // Sov. Phys. Usp. 1972. V. 15. N 2. P. 184-192.

[60] Гинзбург В.Л. Теоретическая физика и астрофизика. Дополнительные главы (2-е издание). Рипол Классик, 1981. Гл. 9. С. 211; Ginzburg V.L. Theoretical physics and astrophysics. Pergamon Press Ltd, 1979. Chapter VIII. P. 171.

[61] Болотовский Б.М., Серов А.В. // УФН. 2005. Т. 175. № 9. C. 943-955; Bolotovskii B.M., Serov A.V. // Phys. Usp. 2005. V. 48. N 9. P. 903-915.

[62] Малыкин Г.Б., Романеи, Е.А. // Опт. и спектр. 2012. Т. 112. № 6. C. 993-1008; Malykin G.B., Romanets E.A. // Opt. Spectrosc. 2012. V. 112. N 6. P. 920-934.

[63] Красильников В.Н., Панкратов А.М. Проблемы дифракции и распространения волн. Л.: ЛГУ, № 8. 1968. С. 59-84.

[64] Розанов Н.Н., Федоров Э.Г., Мацковский А.А. // Квант. электрон. 2016. Т. 46. № 1. С. 13-15; Rosanov N.N., Fedorov E.G., Matskovsky A.A. // Quantum Electron. 2016. V. 46. N 1. P. 13-15.

[65] Розанов Н.Н., Федоров Э.Г. // Опт. и спектр. 2016. Т. 120. № 5. C. 855-859; Rosanov N.N., Fedorov E.G. // Opt. Spectrosc. 2016. V. 120. N 5. P. 803-807.

[66] Сучков А.Ф. // ЖЭТФ. 1965. Т. 49. № 5. С. $1495-$ 1503; Suchkov A.F. // Sov. Phys. JETP. 1965. V. 22. N 5. P. 1026-1031.

[67] Kartashov Y.V., Astrakharchik G.E., Malomed B.A., Torner L. // Nature Reviews. Physics. 2018. V. 20. P. 113019. doi 10.1038/s42254-019-0025-7

[68] Veretenov N.A., Rosanov N.N., Fedorov S.V. // Phys. Rev. Lett. 2016. V. 117. N 18. P. 183901.

[69] Veretenov N.A., Fedorov S.V., Rosanov N.N. // Phys. Rev. Lett. 2017. V. 119. N 26. P. 263901.

[70] Fedorov S.V., Veretenov N.A., Rosanov N.N. // Phys. Rev. Lett. 2019. V. 122. N 2. P. 023903.

[71] Veretenov N.A., Fedorov S.V., Rosanov N.N. // Phil. Trans. R. Soc. A. 2018. V. 376. P. 20170367.

[72] Федоров С.В., Розанов Н.Н., Веретенов Н.А. // Письма в ЖЭТФ. 2018. Т. 107. № 5. С. 342-346; Fedorov S.V., Rosanov N.N., Veretenov N.A. // JETP Lett. 2018. V. 107. N 5. P. 327-331.

[73] Kawauchi A. A Survey of Knot Theory. Basel, Switzerland: Birkhauser, 1996. 